УДК 303 JEL A22, B41, P50

Райченко Александр Васильевич д-р экон. наук, ФГБОУ ВО «Государственный университет управления», г. Москва, Российская Федерация

ORCID: 0000-0001-5776-013X

e-mail:A.V.Raychenko@bk.ru

\section{Raychenko Alexandr}

Doctor of Economic Sciences, State University of Management, Moscow, Russia ORCID: 0000-0001-5776-013X

e-mail: A.V.Raychenko@bk.ru
DOI 10.26425/1816-4277-2020-1-23-27

\section{О СООТНОШЕНИИ ТЕОРИИ И МЕТОДОЛОГИИ УПРАВЛЕНИЯ}

\begin{abstract}
Аннотация. Представлен альтернативный подход к исследованию соотношения методологии и теории управления. Исходя из классического понимания рассматриваемых категорий, предложен новый формат построения научных представлений, в котором теория необходимо основывается на постановке и применении определенной методологии. Представленный формат позволяет не только фундаментально обосновать теорию управления, но и обеспечить ее конструктивное позиционирование в общей системе знаний на основе универсальности методологии. Развитие представленных публикацией исследований, позволит открыть принципиально новые возможности научных обоснований и построений в управлении и других стремительно развивающихся областях знаний и сферах деятельности.
\end{abstract}

Ключевые слова: методология, организация, соотночение, теория, управление, учение.

Цитирование: Райченко А.В. О соотношении теории и методологии управления//Вестник университета. 2020. № 1. С. 23-27.

\section{ON THE CORRELATION OF MANAGEMENT THEORY AND METHODOLOGY}

\begin{abstract}
An alternative approach to the study of the relationship between methodology and management theory has been presented. Based on the classical understanding of the categories under consideration, a new format for constructing scientific representations has been proposed, in which the theory is necessarily based on the formulation and application of a certain methodology. The format presented by the publication allows us not only to substantiate fundamentally the theory of management, but also to ensure its constructive positioning in the general system of knowledge based on the universality of the methodology. The development of the research presented by the publication will enable to discover fundamentally new opportunities for scientific substantiation and construction in management and other swiftly developing areas of knowledge and activities.
\end{abstract}

Keywords: methodology, organization, correlation, theory, management, teaching.

For citation: Raychenko A.V. (2020) On the correlation of management theory and methodology. Vestnik universiteta. I. 1, pp. 23-27. DOI: 10.26425/1816-4277-2020-1-23-27

Классическое словосочетание «теория и методология» устойчиво применяется и традиционно воспринимается большинством исследователей фундаментальных основ управления. Это же словосочетание исчерпывающе определяет формат абсолютного большинства исследований и разработок, представляющихся в монографиях и учебниках, название которых, как правило, с него и начинается. Такое применение настолько консервативно закрепилось в научных подходах и концептуальных построениях, что не вызывает у большинства исследователей практически никаких сомнений [4, с. 7]. Это существенно ограничивает попытки обоснования и реализации альтернативного сочетания и соответствующего ему формата представления фундаментальных основ любой сферы исследований. Вместе с тем содержание и, главное, логика построения инновационных исследований де-факто все чаще рассматривают и практически применяют вариации подобных альтернатив для более эффективного достижения поставленных целей [2, с. 5].

Фундаментально обоснованное понимание методологии как учения об организации деятельности, объединяет «...процедуры, приёмы и методы науки в единую конструктивную программу...» [8]. Это понимание изначально определяется, концептуально позиционируется, адаптивно представляется инструментарием

(C) Райченко А.В., 2020. Статья доступна по лицензии Creative Commons «Attribution» («Атрибуция») 4.0. всемирная (http://creativecommons.org/licenses/by/4.0/).

The Author(s), 2020. This is an open access article under the CC BY 4.0 license (http://creativecommons.org/licenses/by/4.0/). 
познания, проектирования и осуществления как исследований, так и воздействий. В свою очередь, фундаментальное определение теории, как учения о закономерностях исследуемого вида деятельности, изначально представляется, содержательно разрабатывается, последовательно оформляется на основе выбора и применения принятой методологией организации исследований. Этим сравнением обусловливаются достаточно очевидные проявления прямой или косвенной зависимости результатов теоретических построений от выбираемой и применяемой, для их выявления, исследования и представления, методологии. Так, например, опирающиеся на приоритет управления и производность организации исследования изначально меняют местами их объекты и предметы, обусловливая соответствующее представление сущности и содержания закономерностей целенаправленного воздействия [1, с. 275].

Понимание того, что в научно-исследовательской деятельности методология и теория представляют ключевые составляющие любой науки, априори присутствует в большинстве публикуемых научных трудов по управлению [2, с. 7]. Вместе с тем методологии в них нередко отводится чисто символическое значение, напоминающее «ритуальные мантры», не оказывающие существенного воздействия на организацию теоретических изысканий и представление их результатов. С одной стороны, такое позиционирование обусловливает тенденцию проявления чрезмерного субъективизма в исследованиях и представлениях состава и содержания основных понятий и категорий теории. С другой стороны, оно парадоксально определяет изначальный приоритет постановки методологии исследований над выявлением и представлением закономерностей исследуемых процессов и систем. Этому положению находится целый ряд вполне обоснованных подтверждений, которые будут использованы в последующих выводах настоящей публикации.

В фундаментальных научных исследованиях приоритетное позиционирование, конструктивное построение и адаптированное применение методологии необходимо и целенаправленно обеспечивают адекватное обоснование и концептуальное представление теории управления [3, с. 19]. При этом каждая из рассматриваемых составляющих конструктивно основывается, содержательно насыщается, перспективно направляется соответствующим принятой методологии инструментарием. Это обусловлено стратегически ориентированным стремлением открытия неизвестного, разработки инновационного, представления оригинального, решения сложного, достижения желаемого результата деятельности с помощью выстроенного инструментария. С обосновываемых таким образом позиций становится совершенно очевидным, что именно методология определяет формы и методы организации преемственности процесса и пространства инновационных исследований, непрерывного образования и реальной практической деятельности. Очевидно также, что именно она призвана изначально устанавливать, стратегически ориентировать и организационно нацеливать последовательность проведения всего многообразия процедур инновационного познания на поступательное развитие состава и содержания основных концепций теории управления.

Универсальность позиционирования методологии, как учения об организации научных исследований, непрерывного образования, профессиональной деятельности в сфере управления, концептуально определяется, содержательно разрабатывается, логически строится на общей для учебно-исследовательской деятельности модели «исследование - воздействие». В науке управления постановка и применение этой последовательности приобретает особое значение, поскольку в той или иной степени обосновывает, ориентирует и обеспечивает координацию исключительно широкого спектра самых разнообразных видов целенаправленной деятельности. Универсальное значение проявляется в том, как методология управления определяет подходы, концепции, конфигурации анализа, разработки, проектирования, представления и реализации основных положений, целей, задач и путей их решения как в теории, так и на практике. И действительно, в реальной профессиональной деятельности нет социально-экономической организации, которая в той или иной степени не была бы обусловлена каким бы то ни было целеориентирующим, конструктивно определяющим, результативно оптимизирующим управленческим воздействием. Разделяя это положение, необходимо также понимать, что, собственно, такое воздействие и вся совокупность его построений, адаптаций и применений целенаправленно организуются на основе постановки и использования соответствующей методологии исследований и воздействий.

Определение и постановка методологии играют приоритетную роль в организации научных исследований, систематизации ключевых положений, представлении получаемых результатов, обосновании, разработке и внедрении инноваций. Таким пониманием определяется стратегическое значение адекватности постановки и универсальности применения методологии для обоснования и построения основополагающих 
концепций теории управления. Например, всесторонне разрабатываемые в настоящее время программы цифровизации управления изначально обеспечиваются использованием методологических ресурсов, без которых принципиально невозможна их конструктивная реализация. Значение этого настолько велико, что потребовало разработки соответствующей методологии цифровизации управления, все шире обсуждаемой, адаптируемой и используемой в процессах реальных инновационных преобразований в самых разных сообществах [4, с. 121]. Активно расширяющаяся разработка актуальных направлений исследований цифровизации экономики в целом и управления в частности выделяет новые тренды соотношения теории и методологии целенаправленного воздействия. Конструктивный приоритет в них все чаще отдается методологии, как учению об организации исследований и воздействий, закладывающему фундаментальные основы проектирования, разработки и реализации комплекса решаемых при этом задач.

Выделенный выше в настоящей публикации приоритет организации в определении конструктивного соотношения методологии и теории объясняется тем, что именно она устанавливает и классифицирует все многообразие реально рассматриваемых объектов, предметов и методов исследований. С помощью методологии они последовательно изучаются, адекватно оцениваются и целенаправленно используются при разработке и реализации программ цифровизации на единой и, что очень важно, универсальной научной основе. Очевидно, что в таком прикладном формате организации практической деятельности приоритет методологии в рассматриваемом соотношении свойственен практически всем реальным процессам выработки, принятия и реализации целенаправленного воздействия. В процессах реализации и внедрения инноваций он, прежде всего, реализуется штатно обеспечиваемым приоритетом позиционирования этапа организации, необходимо предшествующим последовательности содержательных составляющих исполнения прикладных процедур достижения запланированного. При этом необходимо разделять методологию исследований и организацию управления как целенаправленного воздействия, применяемую в ходе практической деятельности в виде конкретных структур, форм, механизмов и т. п. Вместе с тем актуальная постановка и конструктивное разрешение таких универсально детерминированных по своему повсеместному востребованию задач, реально участвующих в инновациях организаций, также необходимо обеспечивается приоритетом обоснования соответствующих подходов, принципов и других ресурсов методологии управления.

Проведение научно-практических исследований и разработок востребует более гибкий подход к позиционированию их организации в обоснованном выше представлении соотношения методологии и теории управления. Проведенные автором исследования организации руководства и управления в Ракетно-космической корпорации «Энергия» им. С. П. Королёва (далее - РКК «Энергия») показали, что в ходе осуществления таких изысканий, построений и апробаций возникает реальная потребность в трансформации первоначально установленного соотношения теории и методологии управления с целями выделения и разработки самостоятельных задач и решений $[5$, с. 78$]$. Востребованием таких построений выделяется тенденция адаптации вариантов соотношения методологии и теории управления не только в отдельных сферах исследований и видах деятельности, но и в ходе непосредственного анализа и решения достаточно значимых научно-практических задач. И действительно, выделение самостоятельных процессов прикладных разработок по аналогии с известной моделью «цикл в цикле» не только допускает, но и явно востребует оригинальную постановку соотношения методологии и теории применительно к конкретным задачам каждой из таких составляющих. Детализация разнообразия и адаптация применения таких вариаций требует проведения самостоятельного научного исследования, выходящего за рамки настоящей публикации, но уже приведенного выше обоснования достаточно, на взгляд автора, для позиционирования возможности их построения, адаптации и использования в универсальном спектре вариаций соотношения методологии и теории управления.

В прикладных процессах позиционирование методологии как учения об организации адаптации, апробации и использовании знания действия закономерностей, применения принципов, реализации функций и других ключевых составляющих теории управления на практике органично завершает жизненный цикл воздействия. Конструктивность соотношения теории и методологии в этом формате определяется традиционным для понимания большинства исследователей и реальных управляющих естественным и необходимым переходом от теории к практике. С этих позиций вполне допустимым представляется традиционное соотношение теории и методологии, как освоение и использование знания закономерностей. Это знание развивается и реализуется организацией объектной адаптации и прикладного применения фундаментального учения об управлении 
в профессиональной практической деятельности. Более того, традиционный формат рассматриваемого соотношения, в котором методология обеспечивает освоение и использование знаний теории целенаправленного воздействия, применяемое в качестве инструментария их адаптации и реализации, не только сохраняет, но и существенно расширяет свою востребованность в практической деятельности по организации управления.

Представляемые настоящей публикацией положения и выводы исследований автора апробировались и совершенствовались в условиях постановки и разрешения актуальных задач управления такими устойчиво развивающимися организациями, как: Научно-производственное объединение «Слава», Институт повышения квалификации «Машприбор», РКК «Энергия» и другие, обсуждались научным сообществом и практикующими руководителями на профильных форумах и конференциях. Так, прошедшее 20 марта 2019 г. в ФГБОУ ВО «Государственный университет управления» (далее - ГУУ) очередное заседание участников Круглого стола теоретико-методологического кластера ведущих ученых и преподавателей таких вузов, как: ФГБОУ ВО «Московский государственный университет имени М. В. Ломоносова», ФГБОУ ВО «Российская академия народного хозяйства и государственной службы при Президенте Российской Федерации», ФГБОУ ВО «Российский экономический университет имени Г. В. Плеханова», ФГОБУ ВО «Финансовый университет при Правительстве Российской Федерации», и руководящего состава ряда корпораций - выпускников всех уровней образования: Московского инженерно-экономического института имени Серго Орджоникидзе, Московского института управления имени Серго Орджоникидзе, Государственной академии управления имени Серго Орджоникидзе, ГУУ, - предметно рассмотрело и обсудило актуальность обоснования соотношения теории и методологии управления как в учебно-исследовательских, так и в инновационно-внедренческих программах и проектах. В состоявшейся на заседании Круглого стола дискуссии на тему «Фундаментальные основы и инновационные концепции методологии и теории управления» отмечалась актуальность обоснования конструктивности соотношения теории и методологии, как постановочного формата мобилизации и использования наиболее востребованных ресурсов науки управления. Как ведущие исследования теории управления ученые, так и реально участвующие в процессах руководства организациями практики обратили особое внимание на необходимость конкретизации фундаментально обоснованного, исчерпывающего спектра возможных вариаций соотношения методологии и теории как составных частей современной науки управления.

Проведенные апробации положений и результатов, представляемого публикацией исследования, их активное использование в преподавании курсов управленческих дисциплин в ведущих экономических вузах страны, отмеченное в выступлениях Круглого стола 20 марта 2019 г., адаптация и применение положений методологии в практическом управлении организациями сформировало всесторонне обоснованное представление ключевого значения постановки соотношения теории и методологии управления [5, с. 6]. Широкий спектр вариаций: от «методологии и теории» в исследовании и освоении концепций управления, до «теории и методологии» в адаптации и применении положений науки в практическом управлении позволяет универсализировать его постановку и использование. Одним из ключевых для научно-образовательных процессов выводов этого исследования становится утверждение приоритета методологии над теорией, которое находит свое выражение, в спектре других построений и в соответствующем названии учебников и учебных пособий. Очевидным также становится вывод о необходимости освоения методологии исследований и использования положений основополагающих концепций науки управления, необходимо обеспечивающей изучение всего комплекса управленческих дисциплин экономических вузов.

\section{Библиографический список}

1. Девятков, В. В. Методология и технология имитационных исследований сложных систем: учебник. - М.: Инфра-М, 2015. $-448 \mathrm{c}$.

2. Мокий, М. С., Лукьянова, Т. А. Методология научных исследований. Трансдисциплинарные подходы и методы: учебное пособие. - М.: Юрайт, 2019. - 160 с.

3. Новиков, Д. А. Методология управления: учебник. - М.: Либроком, 2016. - 128 с.

4. Овчаров, А. О., Овчарова, Т. М. Методология научного исследования: учебник. - М.: Инфра-М, 2019. - 304 с.

5. Райченко, А. В. Методология опережающего развития в управленческом образовании: монография. - Саарбрюкен: Lambert AP, 2017. - $162 \mathrm{c}$.

6. Райченко, А. В. Постановка методологии цифровизации управления: монография. - М.: Русайнс, 2019. - 182 с. 
7. Фролов, Ю. В. Теория организации и организационное поведение. Методология организации: учебное пособие. - 2-е изд., испр. и доп. - М.: Юрайт, 2019. - 116 с.

8. Большая Российская энциклопедия [Электронный ресурс]. - Режим доступа: https://bigenc.ru/ (дата обращения: 06.11.2019).

\section{References}

1. Devyatkov V. V. Metodologiya i tekhnologiya imitatsionnykh issledovanii slozhnykh system: uchebnik [Methodology and technology of simulation studies of complex systems: a textbook], Moscow, Infra-M, 2015, 448 p.

2. Mokii M. S., Luk'yanova T. A. Metodologiya nauchnykh issledovanii. Transdistsiplinarnye podkhody i metody: uchebnoe posobie [Research methodology. Transdisciplinary approaches and methods: a tutorial], Moscow, Yurait, 2019, 160 p.

3. Novikov D. A. Metodologiya upravleniya: uchebnik [Management methodology: a textbook], Moscow, Librokom, 2016,128 p.

4. Ovcharov A. O., Ovcharova T. M. Metodologiya nauchnogo issledovaniya: uchebnik [Methodology of scientific research: a textbook], Moscow, Infra-M, 2019, 304 p.

5. Raichenko A. V. Metodologiya operezhayushchego razvitiya v upravlencheskom obrazovanii: monografiya [Methodology of advanced development in management education: monography], Saarbrucken, Lambert AP, 2017, $162 \mathrm{p}$.

6. Raichenko A. V. Postanovka metodologii tsifrovizatsii upravleniya: monografiya [Setting the methodology of digitalization management: monography], Moscow, Rusains, 2019, 182 p.

7. Frolov Yu. V. Teoriya organizatsii i organizatsionnoe povedenie. Metodologiya organizatsii: uchebnoe posobie [Organization theory and organizational behavior. Methodology of the organization: a tutorial], 2-e izd., ispr. i dop., Moscow, Yurait, 2019,116 p.

8. Bol shaya Rossiiskaya entsiklopediya [Great Russian Encyclopedia]. Available at: https://bigenc.ru/ (accessed 06.11.2019). 